\title{
DIVERSIDADE DE LEGUMINOSAS NO CERRADO DO MUNICÍPIO DE SÃO JOÃO DO SÓTER, MARANHÃO, BRASIL
}

\author{
Gustavo da Silva Gomes ${ }^{1}$, Guilherme Sousa da Silva², Gonçalo Mendes da \\ Conceição $^{3}$
}

1 Acadêmico do Curso de Ciências Biológicas Licenciatura, da Universidade

Estadual do Maranhão, Caxias-MA, Brasil (gustavogomes@aluno.uema.br)

2 Mestrando do Programa de Pós-graduação em Botânica do Instituto Nacional de Pesquisa da Amazônia-INPA, Manaus-AM, Brasil

3 Professor Dr. do Centro de Estudos Superiores de Caxias/CESC, da Universidade

Estadual do Maranhão/UEMA, Maranhão/Brasil; Programa de Pós-Graduação em Biodiversidade, Ambiente e Saúde/PPGBAS, Caxias-MA, Brasil

Recebido em: 15/04/2017 - Aprovado em: 22/07/2017 - Publicado em: 31/07/2017 DOI: 10.18677/Agrarian_Academy_2017a15

\begin{abstract}
RESUMO
O trabalho teve como objetivo conhecer a diversidade de Leguminosas no Município de São João do Sóter no estado do Maranhão. Foram realizadas expedições mensais no Município para observação, coleta e identificação do material botânico. Para a coleta dos espécimes vegetais no campo deu-se preferência ao estágio reprodutivo das espécies (Floração e Frutificação). Logo após os espécimes coletados e herborizados foram analisados com auxilio de chaves taxonômicas e literatura especializada para identificação ao menor táxon possível. A partir dos dados obtidos foram amostrados 20 espécimes, 15 gêneros e 15 espécies. De todos os espécimes coletadas a Subfamília Papilionoideae foi a que representou mais espécies (7). Quanto à forma de vida, observou se que o hábito de crescimento predominante foi o do tipo erva (8sp). No que diz respeito ao ambiente onde os espécimes foram coletados, observou-se a predominância das espécies nos ambientes de mata de galeria e campo aberto. A partir dos dados obtidos podemos estabelecer que o Município de São João do Sóter possui uma grande diversidade de espécies de leguminosas e o acréscimo de coletas aumentará a diversidade de espécies que é significativa, para a região e consequentemente para o estado do Maranhão.
\end{abstract}

PALAVRAS-CHAVE: Erva, Fabaceae, Papilionoideae.

\section{DIVERSITY OF LEGUMES IN THE CERRADO OF THE MUNICIPALITY FROM SÃO JOÃO DO SÓTER, MARANHÃO, BRAZIL}

\begin{abstract}
The work had how objective know the diversity of legumes in the municipality of São João do Sóter in the state of Maranhão. Were realized monthly expeditions out in the municipality to observe, collect and identify the botanical material. For the collection
\end{abstract}


of the plant specimens in the field preference was given to the reproductive stage of the species (Flowering and Fruiting). Soon after the collected and herborized specimens was analyzed with the aid of taxonomic keys and specialized literature for identification to the smallest possible taxon. Of the the obtained data were sampled 20 specimens, 15 genera and 15 species. Of all the specimens collected the Subfamily Papilionoideae was the one that represented more species (7). As for the way of life, it was observed that the predominant habit of growth were herb (8sp). Regarding the environment where the specimens were collected, the predominance of the species in gallery forest and open field environments was observed. From the data obtained can establish that the Municipality of São João do Sóter has a great diversity of legume species and the increase of collections will increase the diversity of species that is significant for the region and consequently for the state of Maranhão.

KEYWORDS: Herb, Fabaceae, Papilionoideae.

\section{INTRODUÇÃO}

Leguminosae ou Fabaceae constitui uma das maiores famílias de Angiospermas do mundo, distribuídas em todo o globo, sendo reconhecidos cerca de 727 gêneros e 19.325 espécies (LEWIS et al., 2005). É considerada terceira maior família de angiospermas do planeta e a maior família botânica do Brasil, ocorrendo 2.826 espécies (1.524 endêmicas) agrupadas em 222 gêneros, encontradas na maioria das regiões e distribuídas por quase todas as formações vegetacionais (LIMA 2000; BFG, 2015). Trabalhos florísticos e taxonômicos indicam as Leguminosas como uma das famílias mais representativas em formações florestais (OLIVEIRA-FILHO et al., 1994; RIBEIRO, 1998; LIMA, 2000). Apresentam grandes índices de diversificação, tal como, sua ecologia e morfologia, com hábito de grandes arvores à ervas (LEWIS, 1987; LEWIS et al., 2005).

Tradicionalmente as Leguminosas eram tratadas como apenas um grupo por BENTHAM (1865) formando uma única família contendo três subfamílias (Caesalpinioideae, Mimosoideae e Papilionoideae). Posteriormente HUTCHINSON (1964) e CRONQUIST (1981) propuseram que estas subfamílias fossem consideradas como famílias independentes. De acordo com APG IV (2016), atualmente as subfamílias são consideradas integrantes de uma única família (Fabaceae ou Leguminosae), posicionada na ordem Fabales, do clado Eurosids.

Leguminosae é uma família de grande importância econômica (OKIGBO, 1977), sendo utilizada no forrageamento, marcenaria, como forma medicinal, combustível, pesticida, corante, goma, óleo ou ornamental (DATE, 1977; POLHILL \& RAVEN, 1981; BRANDÃO,1992). Apresentam grande importância ecológica, pois estão bem adaptadas à primeira colonização e exploração de diversos ambientes, por causa de suas associações com bactérias fixadoras de nitrogênio ou com ectomicorrizas. Bactérias do gênero Rhizobium, localizadas em nódulos radiculares encontrados em muitas espécies, convertem o nitrogênio atmosférico em amônia, forma solúvel que pode ser utilizada por 20 outros vegetais, resultando em espécies extremamente valiosas como fornecedores de adubos naturais (LEWIS, 1987).

As leguminosas são culturas alimentares importantes que fornecem fontes altamente nutritivas de proteínas e micronutrientes que podem beneficiar a saúde e os meios de subsistência, particularmente nos países em desenvolvimento (YAHARA et al., 2013). 
O Cerrado é a savana mais rica em biodiversidade no planeta, abrigando $5 \%$ de todas as espécies no mundo e $30 \%$ das espécies do país. Dados estimam que esse bioma possui cerca de 14 mil espécies de plantas (WWF, 2015). O termo Cerrado é geralmente utilizado para designar o conjunto de ecossistemas (savanas, matas, campos e matas de galeria) que ocorrem no Brasil Central (EITEN, 1977; RIBEIRO et al., 1999). Para o estado do Maranhão há uma grande relevância em questão de diversidade para estudos das leguminosas, por possuir grande extensão do domínio fitogeográfico cerrado. O domínio Cerrado possui 51 formações florestais com extensões significativas (destacando-se as florestas estacionais deciduais e em menor abrangência florestas ombrófilas densas), além de uma ampla área de tensão ecológica com o bioma Amazônico (MARANHÃO, 2011).

O cerrado apesar de possuir grande biodiversidade e ser o centro de grandes enfoques de estudo, observa-se que ainda há poucos trabalhos sobre a família Fabaceae. Nesse aspecto, há uma necessidade de estudos sobre a flora do estado e principalmente do município de São João do Sóter, levando em conta a existência do domínio Cerrado com grande potencial de ocorrência dessa família. A execução dessa pesquisa teve como objetivo conhecer a diversidade de Leguminosas do Cerrado no Município de São João do Sóter, Maranhão, onde aspectos florísticos e taxonômicos fornecerão base para futuras pesquisas das mais diversas naturezas a respeito dessa família, visando resolver questões relacionadas à grande complexidade da nomenclatura vegetal.

\section{MATERIAL E MÉTODOS}

O município de São João do Sóter está localizado no Estado de Maranhão, se estende por $1.438,1 \mathrm{~km}^{2}$ e conta com 17.238 habitantes no último censo realizado. A densidade demográfica é de 11,9 habitantes por $\mathrm{km}^{2}$ no território do município. São João do Sóter se situa a $57 \mathrm{~km}$ a Sul-Oeste de Caxias a maior cidade nos arredores. Situado a 108 metros de altitude, de São João do Sóter as coordenadas geográficas do município são: $5^{\circ} 6^{\prime} 28^{\prime \prime} \mathrm{S}$. e $43^{\circ} 48^{\prime} 34^{\prime \prime} \mathrm{W}$ (Fig. 1). $\mathrm{O}$ Município foi criado pela Lei № 6.157, de 10 de novembro de 1994 (IBGE, 2012).

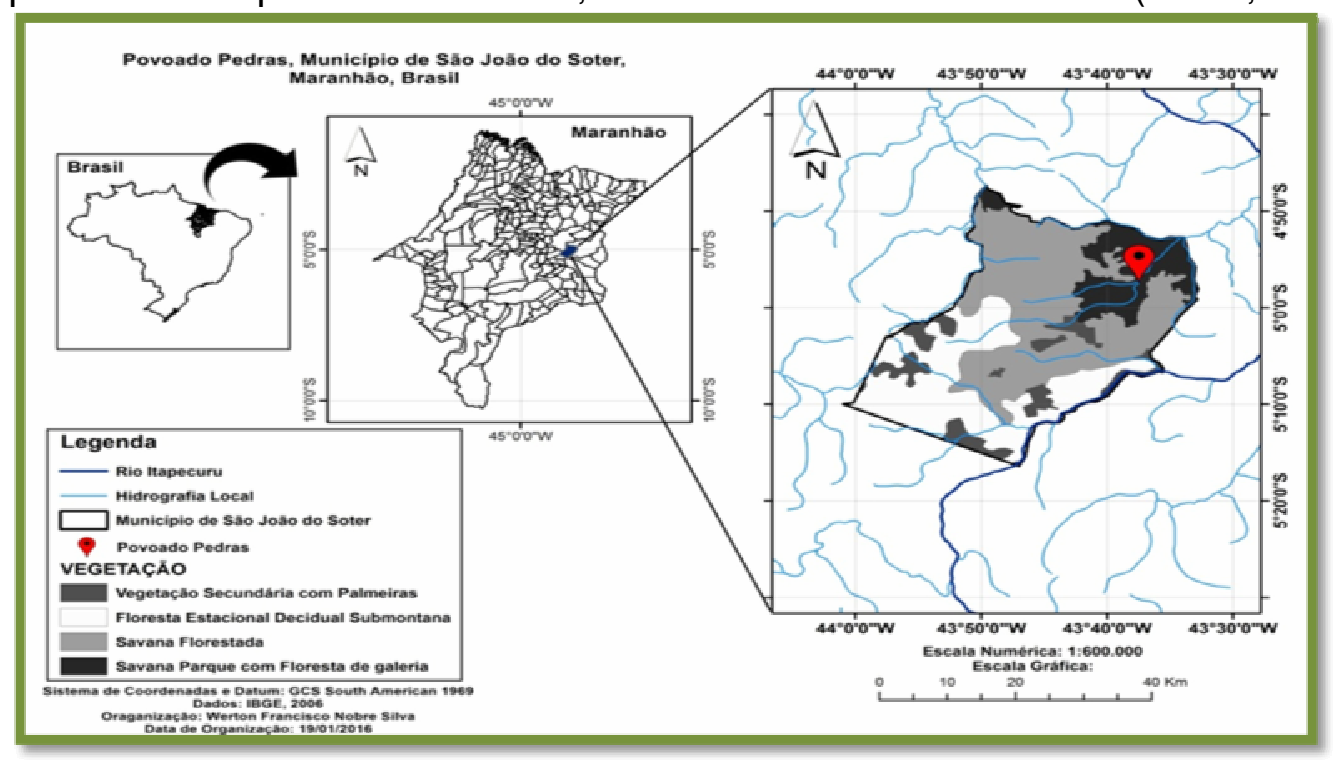

FIGURA 1. Mapa do Município de São João do Sóter, Maranhão. Fonte: IBGE; 2006, Google Earth 2014, Organização: SILVA, (2015). 
O levantamento florístico compreendeu três etapas: o trabalho em campo, preparação e análise e a identificação desse material coletado. A coleta do material botânico foi realizada durante o período seco compreendendo os meses de outubro a novembro de 2016, onde visitou-se o município de São João do Sóter.

Para o trabalho de campo, com o auxílio de tesoura de poda foram coletados os espécimes em estado fértil, obtendo no mínimo cinco ramos de cada indivíduo da área, que foram acondicionados em saco plástico e etiquetados de acordo com a sequência de coleta (Fig. 2). Para cada espécime coletado foi realizada uma serie de anotações como: data e local de coleta (localidade, município, estado e coordenadas geográficas), o nome do coletor, e as características físicas do ambiente (como declividade, tipo do solo, exposição, proximidades de cursos d'água etc.;).

As características da planta e da coleta propriamente dita foram anotadas em caderno de campo: o hábito da planta, altura, característica da casca, cor e textura das folhas, flores e frutos e outras características marcantes que auxiliaram na hora da identificação. Após a coleta, o material botânico foi herborizado sendo processado da seguinte forma: os espécimes vegetais foram prensados entre folhas de jornal sendo estes acoplados com papelão e uma estrutura de madeira e amarrados em uma corda sendo exposta a temperatura ambiente. Para identificação os espécimes coletados foram identificados em família, gênero e espécie, levando em consideração comparação com material especializado, tais como bibliografias, artigos científicos, livros, herbários virtuais e chaves taxonômicas, sendo confirmadas por especialista.

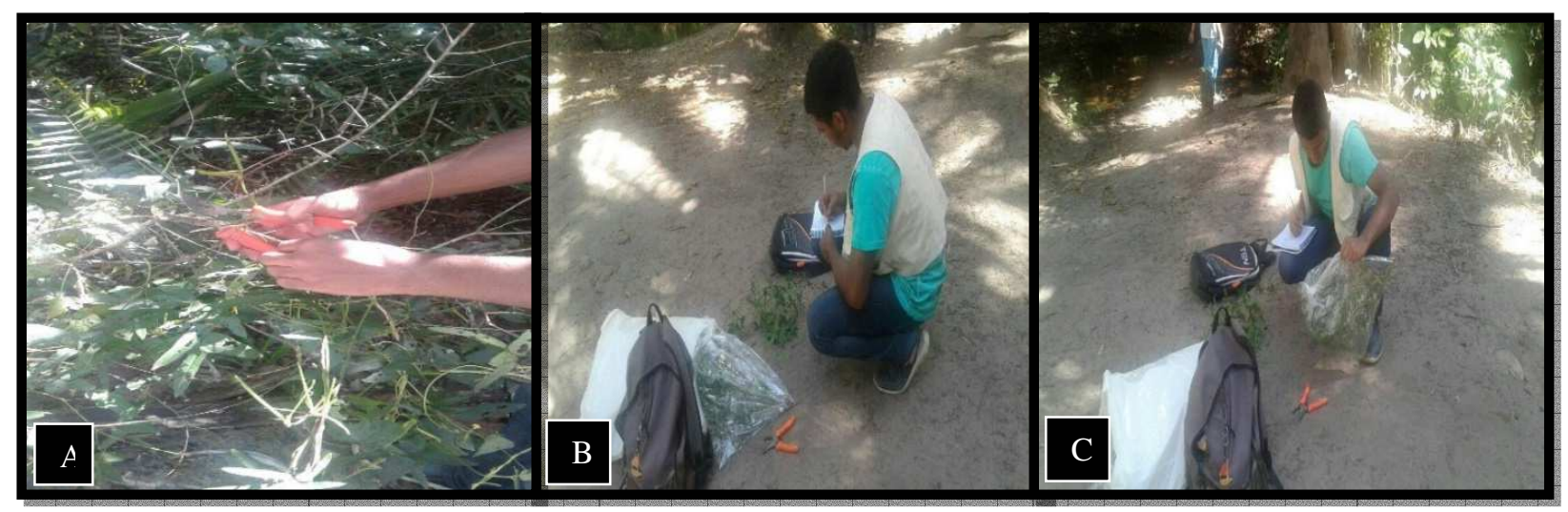

FIGURA 2. Procedimentos Metodológicos. A) Coleta do Material Botânico; B) Anotações em Campo; C) Acondicionamento do material botânico. Fonte: SILVA (2016).

\section{RESULTADOS E DISCUSSÃO}

Foram registradas para a área de estudo 15 espécies e 15 gêneros (Quadro 1). As três subfamílias foram amostradas no presente levantamento, o que demonstra um indicativo da diversidade das leguminosas para a área. 
QUADRO 1. Lista de espécies de Fabaceae e subfamílias registradas para o município de São João do Sóter/Maranhão.

\begin{tabular}{|c|c|c|}
\hline $\mathbf{N}^{\circ}$ & Subfamília & Espécie/ Autor \\
\hline 1 & \multirow{6}{*}{ CAESALPINOIDEAE } & Bauhinia dubia G.Don \\
\hline 2 & & Phanera glabra (Jacq.) Vaz \\
\hline 3 & & Plathymenia reticulata Benth. J. Bot. \\
\hline 4 & & Senegalia poliphyla (DC) Briton. \\
\hline 5 & & $\begin{array}{l}\text { Senna reticulata (Willd.) H.S. Irwin \& } \\
\text { Barneby }\end{array}$ \\
\hline 6 & & Tamarindus indica $\mathrm{L}$. \\
\hline 7 & \multirow{7}{*}{ PAPILIONOIDEAE } & Aeschymoneme histrix Poir. \\
\hline 8 & & Centrosema brasiliana (L.) Benth. \\
\hline 9 & & Dioclea bicolor Benth. \\
\hline 10 & & Phaseolus vulgaris $\mathrm{L}$. \\
\hline 11 & & Periandra heterophyla Benth. \\
\hline 12 & & Crotalaria retusa L. \\
\hline 13 & & Pterodon emarginatus Vogel. \\
\hline 14 & \multirow[t]{2}{*}{ MIMOSOIDEAE } & Mimosa caesalpinifolia Benth. \\
\hline 15 & & Mimosa sensitiva L. \\
\hline
\end{tabular}

A subfamília Papilionoideae foi a mais representativa entre as subfamílias amostradas (Fig. 3). Papilionoideae é a maior das Leguminosas, essa tem como característica plantas herbáceas comuns em regiões temperadas, e em regiões tropicais, tendo um maior numero de espécies lenhosas (BARROSO et al., 1999). Papilionoideae tem características marcantes como folhas imparipinadas, trifolioladas ou unifolioladas; flores diclamídeas, corola com prefloração imbricada descendente ou vexilar, dialipétala, estames geralmente em número duplo ao das pétalas, sendo frequentemente nove estames unidos entre si e um livre ou todos unidos, não vistosos e sementes sem pleurogama, mas com hilo bem desenvolvido (SOUZA \& LORENZI, 2005). 


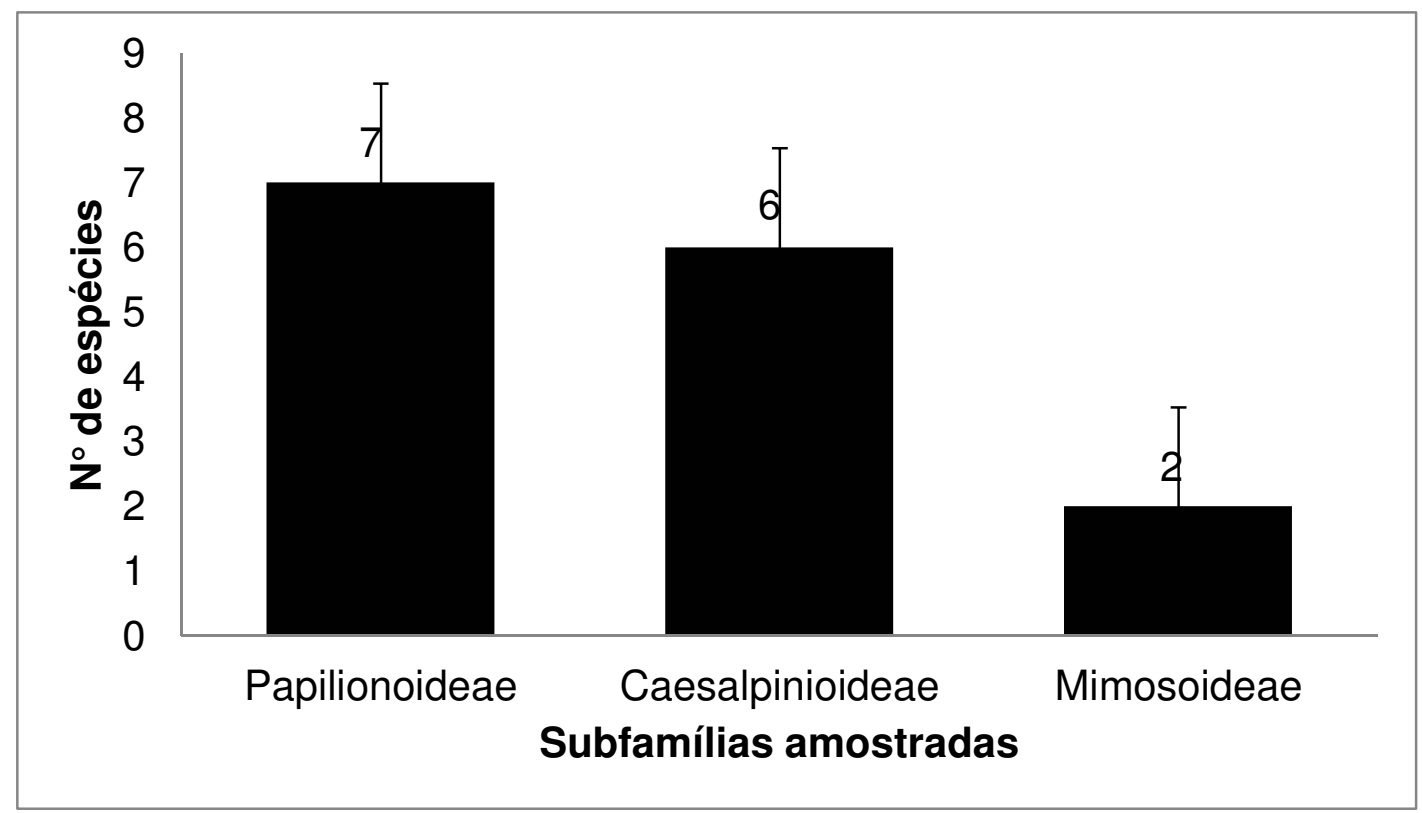

FIGURA 3. Representação da quantidade de espécies separadas por subfamília coletadas no município de São João do Sóter, Maranhão. Brasil.

Quanto a Forma de vida, observou se que o hábito de crescimento predominante foi o do tipo erva, com oito sp (Fig. 4). Segundo a FLORA DO BRASIL (2017) para o Brasil são estimados que existam cerca de 352 espécies da família Fabaceae com esse tipo de hábito de crescimento o que demonstra a grande diversidade da família quanto ao tipo de vida. O hábito dessa família é variado, desde arvores, arbustos, subarbustos até ervas eretas, rastejantes, ou inda trepadeiras. (JUDD et al. 1999).

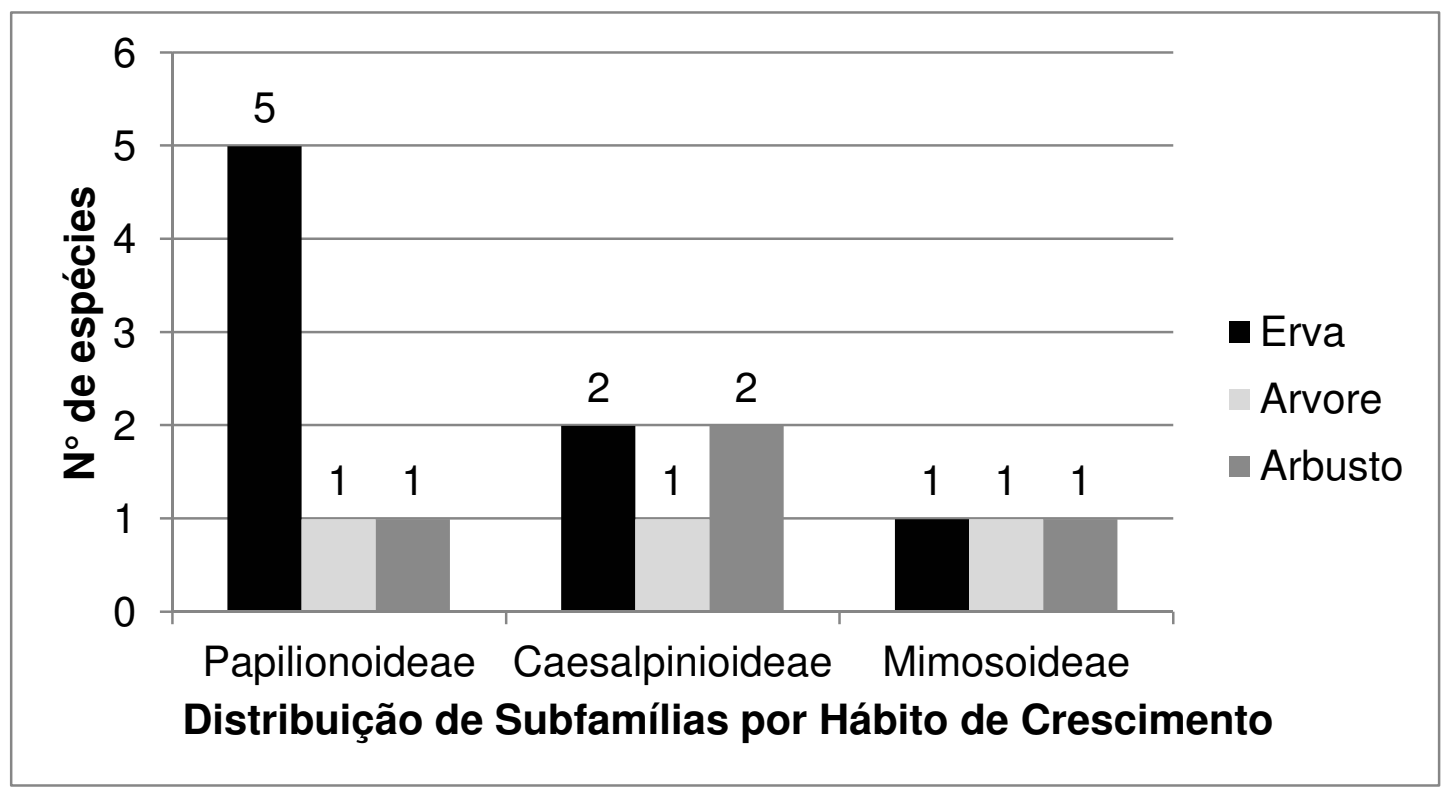

FIGURA 4. Distribuição das três subfamílias de Leguminosae de acordo com o Hábito de Crescimento no município de São João do Sóter, Maranhão, Brasil. 
No que diz respeito ao ambiente onde os espécimes foram coletados, observou-se a predominância das espécies nos ambientes de mata de galeria e campo aberto (Fig. 6). Os ambientes onde foram coletados os espécimes apresentavam claramente atividades antropogênicas, tais como, queimadas e corte da vegetação.

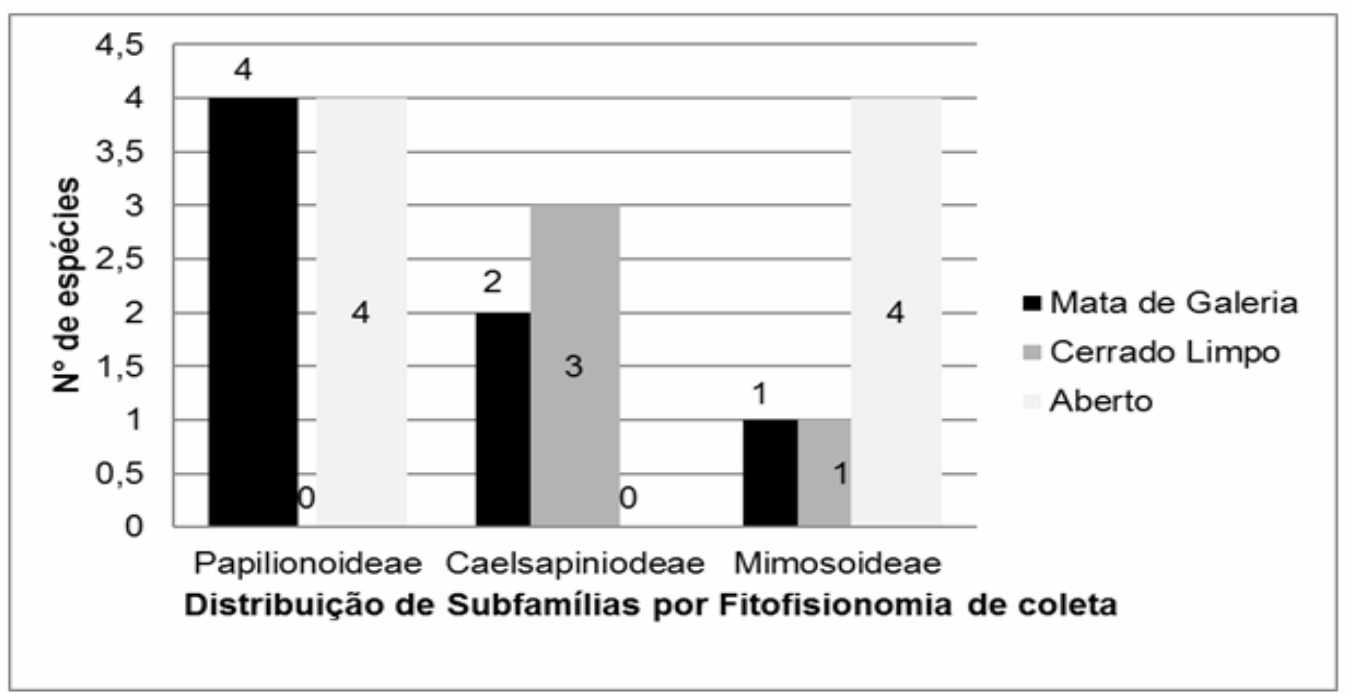

FIGURA 5. Representação dos ambientes de coleta das subfamílias Fabaceae nos ambientes de coleta

Fabaceae é uma família bem extensiva, e o cerrado é um dos fatores que justifica essa grande expansão de espécies e pela grande variação na fisionomia, apresentando formas florestas, savânicas e campestre que fazem com que as espécies da família consigam manter-se e reproduzir (RIBEIRO \& WALTER, 1998). O Cerrado ocupa cerca de $23 \%$ do território brasileiro, é um bioma considerável por sua grande variação fitofisionômica (EITEN, 1972). De acordo com CORDEIRO (2000), Fabaceae, por possuir a capacidade de nodulação de suas espécies, apresenta maior aclimatação em regiões com baixo teor de nitrogênio, sendo essa uma vantagem sobre as demais espécies, principalmente no Cerrado, onde o solo, na maioria das vezes, é pobre em nutrientes.

\section{CONCLUSÃO}

Foram amostrados 15 espécies e 15 gêneros, com todas as subfamílias amostradas. A subfamília Papilionoideae foi a que apresentou mais espécies, das 15 identificadas. Quanto ao hábito de crescimento Erva foi o predominante. No que diz respeito ao ambiente onde os espécimes foram coletados, observou-se a predominância das espécies nos ambientes de mata de galeria e campo aberto.

No total foi amostrado uma diversidade significativa, demonstrando uma quantidade relevante, levando em consideração o grande potencial que o município de São João do Sóter tem em riqueza de Fabaceae. A partir dos dados obtidos pode-se estabelecer que o município possui uma grande diversidade de espécies para a família, e que o acréscimo de coletas aumentará a diversidade de espécies que já se demonstra significativa. 


\section{REFERÊNCIAS}

APG. The angiosperm phylogeny group. An update of the Angiosperm Phylogeny Group classification for the orders and families of flowering plants: APG IV. Botanical Journal of the Linnean Society, v. 181, p. 1-20, 2016.

BARROSO, G. M.; MORIM, M. P.; PEIXOTO, A. L.; ICHASO, C. L. F. Frutos e sementes: morfologia aplicada à sistemática de dicotiledôneas. Editora UFV, Viçosa, p. 443. 1999.

BENTHAM, G. Leguminosae. In: BENTHAM, G.; HOOKER, J. D. Sistens dicotyledonum polypetalarum ordines XI: Leguminosas-Myrtaceaeas. Genera Plantarum. London: Lovell Reeve e Co, v. I, n. 2, p. 434-600, 1865.

BFG - The Brazil Flora Group. Growing knowledge: an overview of Seed Plant diversity in Brazil. Rodriguésia, v.66, n.4, p.1085-1113. 2015.

BRANDÃO, M. Plantas Forrageiras do Cerrado. Revista Informe Agropecuário. v. 16, p. 36-39, 1992.

IBGE. Censo Cidades. Instituto Brasileiro de Geografia e Estatísticas. 2012. Disponível em: https://cidades.ibge.gov.br/v4/brasil/ma/sao-joao-do-soter/panorama Acesso em: 06/07/2017

CORDEIRO, L. Fixação de nitrogênio em leguminosas ocorrentes no cerrado. In: KLEIN, A. L. Eugen Warming e o Cerrado brasilleiro: um século depois. Universidade Estadual de São Paulo. São Paulo, p.131-145. 2000.

CRONQUIST, A. An integrated system of classification of flowering plants. Columbia University Press, New York. p. 1262, 1981.

DATE, R. A. The development and use of Legume inoculants. In: AYANABA, A.; DART, P. J. Biological nitrogen fixation in farming systems of the tropics. John Wiley \& Sons Ltda. New York, p. 169-180, 1977.

EITEN, G. Delimitação do conceito de Cerrado. Arquivos do Jardim Botânico, Rio de Janeiro v.21, p. 125-134, 1977.

EITEN, G. The cerrado vegetation of Brazil. Botanical Review. v. 38, n. 2, p.201$341,1972$.

FLORA DO BRASIL, Lista de Espécies da Flora do Brasil. Jardim Botânico do Rio de Janeiro. 2017. Disponível em: <http://floradobrasil.jbrj.gov.br/>. Acesso em: 23/06/2017.

HUTCHINSON, J. The genera of flowering plants. Oxford University Press, Oxford. v. 1. p. $516,1964$. 
JUDD, W. S.; CAMPBELL, C. S.; KELLOG, E. A.; STEVENS, P. F.; DONOGHUE, M. J. Plant systematics: a phylogenetic approach. Suderland: Sinner Associates. p. 464, 1999.

LEWIS, G. P. Legumes of Bahia. Royal Botanic Gardens, Kew. p. 369, 1987.

LEWIS, G. P.; SCHRIRE, B. D.; MACKINDER, B. A.; LOCK, J. M. Legumes of the World. Royal Botanic Gardens, Kew. p. 577, 2005.

LIMA, H. C. Leguminosas arbóreas da Mata Atlântica - uma análise da riqueza, padrões de distribuição geográfica e similaridades florísticas em remanescentes florestais do estado do Rio de Janeiro. Tese de Doutorado. Universidade Federal do Rio de Janeiro, Rio de Janeiro, p.122, 2000.

MARANHÃO. Plano de Ação Para Prevenção e Controle do Desmatamento e das Queimadas no Estado do Maranhão. Governo do Estado do Maranhão. Secretaria de Estado do Meio Ambiente e Recursos Naturais. p. 110, 2011.

OKIGBO, B. N. Legumes in farming systems of the humid tropics. In: AYANABA, A.; DART, P. J. Biological nitrogen fixation in farming systems of the tropics. John Wiley \& Sons Ltda. New York, p. 61-72. 1977.

OLIVEIRA-FILHO, A. T.; VILELAS, E. A.; GAVILANES, M.L.; CARVALHO, D. A. Comparison of the flora and soils of six areas of Montane Semideciduous Forest in southern Minas Gerais, Brazil. Endinburgh Journal of Botany. v. 51, n. 3, p. 355389, 1994.

POLHILL, R. M; RAVEN, P. H. Advances in Legume Systematics. Royal Botanic Garden, Kew. v. 1, p. 425, 1981.

RIBEIRO, J. F. Cerrado: Matas de galeria. Embrapa-CPAC, Planaltina, p.164, 1998.

RIBEIRO, J.E. L. S.; HOPKINS, M.J.G.; VICENTINI, A.; SOTHERS, C.A.; COSTA, M. A. S.; BRITO, J. M.; SOUZA, M.A. D.; MARTINS, L.H.P.; LOHMANN, L.G.; ASSUNÇÃO, P.A.C.L.; PEREIRA, E. C.; SILVA, C. F.; MESQUITA, M.R.; PROCÓPIO, L.C. Flora da Reserva Ducke: Guia de identificação das plantas vasculares de uma floresta de terra-firme na Amazônia Central. INPA, Manaus. p. 816. 1999.

RIBEIRO, J. F.; WALTER, B.M.T. Fitofisionomias do Bioma Cerrado. In: SANO, S.M.; ALMEIDA, S.P. (eds.). Cerrado, ambiente e flora. Embrapa - CPAC, Planaltina. p. 89-166, 1998.

SOUZA, V. C.; LORENZI, H. Botanica Sistemática: Guia llustrado para Identificação das Famílias de Angiospermas da Flora Brasileira. Baseado em 
APG II. .1. ed. Nova Odessa: Instituto Plantarum de Estudos da Flora Ltda, p. 640, 2005.

SILVA, F. W. N. Mapa do Município de São João do Sóter, Maranhão. 2015. IBGE; 2006, Google Earth 2015.

WWF-Brasil. Áreas Prioritárias para Conservação da Biodiversidade no Cerrado e Pantanal. Brasília-DF, 2015: Disponível em http://d3nehc6yl9qzo4.cloudfront.net/downloads/publicacao_areasprioritarias_cerrad o_pantanal_1.pdf. Acesso em 17/01/2017.

YAHARA, T.; JAVADI, F.; ONODA, Y.; QUEIROZ, L. P.; FAITH, D. P.; PRADO, D. E.; AKASAKA, M.; KADOYA, T.; ISHIHAMA, F.; DAVIES, S.; SLIK, J. W. F.; YI, T.; MA, K.; BIN, C.; DARNAEDI, D.; PENNINGTON, R. T.; TUDA, M.; SHIMADA, M.; ITO, M.; EGAN, A. E.; BUERKI, S.; RAES, N.; KAJITA, T.; VATANPARAST, M.; MIMURA, M.; TACHIDA, H.; IWASA, Y.; SMITH, G. F.; VICTOR, J. E.; NKONKI, T. Global legume diversity assessment: Concepts, key indicators, and strategies. Taxon. v. 62, p. 249-266, 2013. 